\title{
NURBS Fitting Algorithm of Section Contour Data based on Two-photon Polymerization Process
}

\author{
Yucheng $\mathrm{Li}^{\mathrm{a}}$, Jieqiong $\mathrm{Lin}^{\mathrm{b}}{ }^{*}$, Baojun $\mathrm{Yu}^{\mathrm{c}}$ and Xian Jing ${ }^{\mathrm{d}}$ \\ School of Mechatronic Engineering, Changchun University of Technology, Changchun \\ 130000,China; \\ ajilinliyucheng@sina.com, ${ }^{b}$ linjieqiong@mail.ccut.edu.cn, ${ }^{c}$ bruce5257@126.com, \\ dzzx_bjx@126.com \\ Corresponding Author: Jieqiong Lin
}

Keywords: two-photon polymerization, STL format, NURBS curve, data fitting

\begin{abstract}
Two-photon polymerization is considered to be one of the most promising technique, which could achieve the structured process of three-dimensional micro-nano structure fuctional device. In order to improve the machining precision and machining efficiency of formed parts, in the process of two-photon polymerization, it was necessary to file the slice data. In the process of three-dimensional modelling, because of the NURBS curve has flexibility and local control, it was very suitable to fit the discrete data. Using line and non-uniform rational B-spline curves to fit the sliced data points, and according to the algorithm which based on the judgment of slope and length, the datas were processed by segmented fitting. Moreover, through the dichotomy to optimize the control points to make the intersection contour more precise and smoother.
\end{abstract}

\section{Introduction}

Compared with other microfabrication technology, two-photon polymerization has the characteristics of high precision, good spatial selectivity and true three-dimensional processing ability[1]. In the two-photon polymerization processing, raster scan and contour scan are usually used[2,3]. Compared with the raster scan, contour scan has higher precision and higher machining efficiency, it had been widely used in the scholars. For the contour scan, the fitting of section contour data plays a decisive role in forming precision of final parts. So the research of section contour data fitting has great significance.

Dong Tao and others fitted the section contour data by using the optimal double circular arc fitting technology, and get the high precision and smoothness[4]. According to reduce the influence of step effect, Zhao Jibin and others proposed to fit the edges of Triangle patches into cubic B-spline curve or quadratic curve. Then using NURBS curves to fit the cross section data points.But this method follow-up without detailed[5]. Zhong Shan and others used Clothoid curve fitting algorithm with curvature continuous properties to reconstruct the section contour,and improved the surface quality of forming parts[6]. Therefore, using line and non-uniform rational B-spline curves to fit the sliced data points. What's more, according to the actual situation of the sliced data points to subsection process.

\section{Subsection processing of the section contour data points}

After slicing the STL data model, it will get a series of uneven sliced data points. As shown in Fig.1, BC and DA segment data points are sparse, AB and CD segment data points are more intensive. Therefore, according to the characteristics of data points, segment the data points of the section contour, and according to the curves and straight lines to fit. 


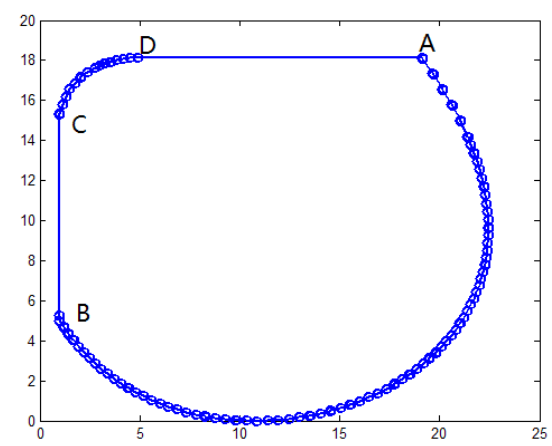

Fig.1 Sketch map of section contour

In this paper, the data points are segmented according to the judgement of slope and length. Calculating the slope of line segment between two adjacent relations. When the relations between the two lines into the vertical or collinear, the data points do not need to fit with NURBS; when as a certain angle $\alpha$, we need to set a threshold data $\alpha_{\max }$. When $\alpha>\alpha_{\max }$, the data points need to subsection process.

When it can not fully explain that the relationship between data points is curve. Thus on the basis of the judgment of slope, it also need the judgment of length. The lines formed by sliced data points, $\left|q_{k-1} q_{k}\right|$ and $\left|q_{k} q_{k+1}\right|$ are meet:

$$
\left|q_{n} q_{n+1}\right|<\delta_{\max },(n=0,1, \mathrm{~L}, m-1)
$$

Therefore, if the data points which consist of the lines do not meet the judgment of slope but meet the judgment of length, these data points can be fitted by NURBS curves. Then other data points are stored according to the original contour.

\section{The design of NURBS curves fitting algorithm}

NURBS curves formula as shown below:

$$
p(u)=\frac{\sum_{i=0}^{n} N_{i, p}(u) \omega_{i} D_{i}}{\sum_{i=0}^{n} N_{i, p}(u) \omega_{i}}=\sum_{i=0}^{n} R_{i, p}(u) D_{i}
$$

$\mathrm{n}$ is the number of control points, the total number is $(\mathrm{n}+1) ; N_{i, p}(u)$ is B-spline base function of $\mathrm{p}$ of NURBS curves; $D_{i}$ are the control points of NURBS curves; $\omega_{i}$ are the weights of control points $D_{i} ; R_{i, p}(u)$ are the rational basis functions of NURBS curves.

In this paper, using the weights of control points as 1[7]. Therefore, NURBS curves are converted to B-spline curves:

$$
p(u)=\sum_{i=0}^{n} N_{i, p}(u) D_{i}
$$

The data points are used to fit by the least squares:

(1) Set $\mathrm{p}$ and $\mathrm{n}$;

(2) $p\left(u_{0}\right)=D_{0}=q_{0}, \quad p($ 荘 $)=D_{n}=q_{m}$;

(3)The article adopt the centripetal parametric method to solve $\stackrel{\circ}{u}_{k}$ [8].

$$
\stackrel{\circ}{u_{k}}=u_{k-1}^{2}+\frac{\left(\left|q_{k}-q_{k-1}\right|\right)^{1 / 2}}{d}, k=1, \mathrm{~L}, m-1
$$

Among them $\stackrel{\circ}{u}_{0}=0, \mathbb{Z}_{m}=1$, d indicates the fomula of the total chord length, $d=\sum_{k=1}^{m}\left(\left|q_{k}-q_{k-1}\right|\right)^{1 / 2}$. 
(4) With the following method to determine the nodes value[9]. $u_{p+j}=(1-\alpha) \stackrel{2}{u}_{s-1}+\alpha \dot{u}_{s}, j=1,2, \mathrm{~L}, n-p$

Among them $\alpha=j a-s, s=\operatorname{int}(j a)$, $a$ is a positive real number, $a=\frac{m+1}{n-p+1}$, int $(g)$ represents integral function, rounding down to the nearest integer function.

(5) Based on the least square method to get the function for curve fitting, the need to meet:

$$
E_{k}=\sum_{k=0}^{m}\left|q_{k}-p\left(u_{k}\right)\right|^{2}=\sum_{k=0}^{m}\left|q_{k}-\sum_{i=0}^{n} N_{i, p}\left(\dot{u}_{k}\right) D_{i}\right|^{2}
$$

Among them $R_{k}=q_{k}-N_{0, p}\left(\begin{array}{l}\circ \\ u_{k}\end{array}\right) q_{0}-N_{n, p}\left(u_{k}^{\circ}\right) q_{m},(k=1,2, \mathrm{~L}, m-1)$.

According to take the derivative of equation(6) to obtain a linear equation group:

$$
\left(N^{T} N\right) D=R
$$

$\mathrm{N}$ is a $(m-1) \times(n-1)$ matrix consisting of B-spline basis functions. The control points $D_{j}$ will be obtained by Gauss elimination method.

\section{Degree of B-spline basis function and control points $n$}

In the last section NURBS curve is converted into B-spline curve. Cubic B-spline curve second derivative continuous, that is to say, the second order smoothness, so the article makes $p=3[10]$. For the determination of the number of control vertices, the dichotomy strategy is adopted[7]. In determining the minimum number of control points and the maximum number of control points. Use $n_{\text {now }}=\frac{n_{\min }+n_{\max }}{2}$ to fit the data points. According to the fitted maximum error value is consistent with the allowable error or not and then decide whether to re select the two sub range. So repeatedly, we could determine the optimal control points n ultimately and reduce the iteration time.

\section{Instance specification}

STL data model is sliced by the slicing software Magics, The bounding box of the model in Fig.2 is $20.000 \mathrm{~mm} \times 10.000 \mathrm{~mm} \times 2.000 \mathrm{~mm}$, consist of 188 small triangles the model for 50 layer thickness.

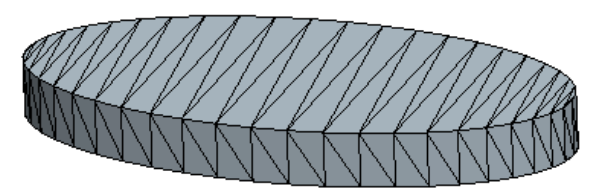

Fig.2 3D sketch map of STL data model

Using the No.31 section contour as an example. Fig.3(a) is the data before fitting, Fig.3(b) is the data of fitted and segmentation points of No.31 layer. 


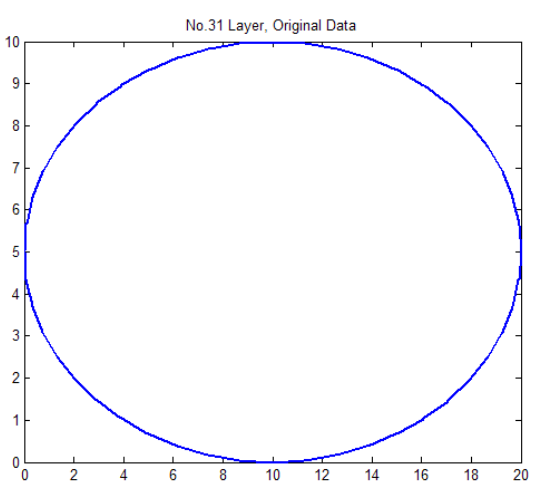

(a) Layered section of No.31 layer

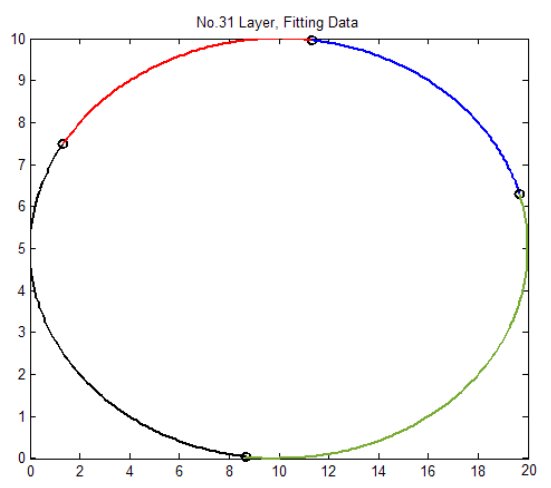

(b) Fitting section of No. 31 layer

Fig.3 Sliced data points processing

Using the algorithm of the data fitting, the allowable error is set to $0.005 \mathrm{~mm}$. After fitting, the original 51 small straight segments are fitted into 4 NURBS curves, The error chart is shown in Fig.4. Tab.1 is the data analysis before and after fitting the section contour of No.31 layer.

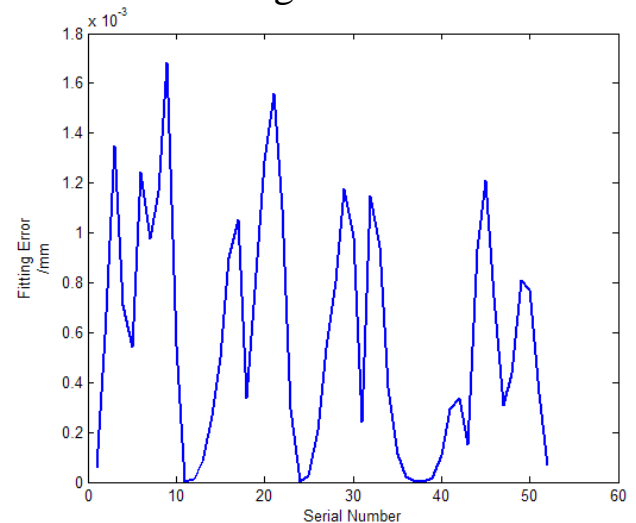

Fig.4 Curve fitting error of No.31 layer

Tab.1 Comparison of the NO.31 layer section contour fitting

\begin{tabular}{cccccc}
\hline $\begin{array}{c}\text { Number of } \\
\text { fitting } \\
\text { segment }\end{array}$ & $\begin{array}{c}\text { Number } \\
\text { of path } \\
\text { segment }\end{array}$ & $\begin{array}{c}\text { Maximum } \\
\text { path } \\
\text { length/mm }\end{array}$ & $\begin{array}{c}\text { Average } \\
\text { path length } \\
\text { /mm }\end{array}$ & $\begin{array}{c}\text { Maximum } \\
\text { fitting } \\
\text { error/ } \mu \mathrm{m}\end{array}$ & $\begin{array}{c}\text { Average } \\
\text { fitting } \\
\text { error/ } \mu \mathrm{m}\end{array}$ \\
\hline $\begin{array}{c}\text { Before } \\
\text { fitting }\end{array}$ & 51 & 1.2756 & 1.0086 & 0.0 & 0.0 \\
After fitting & 4 & 14.0690 & 11.9304 & 1.7000 & 0.9800 \\
\hline
\end{tabular}

\section{Summary}

In view of the causes of the error result of the STL data model section contour, using linear and non-uniform rational B-spline curve to fit the sliced data points. In the process of curve fitting, in order to achieve a better fitting effect, using line and non-uniform rational B-spline curves to fit the sliced data points and optimize the number of control points. At last, the example example analysis shows that, the method is not only express the figure in fewer splines curve, but also improve the precision and smoothness of the section contour within the scope of allowable error.

\section{Acknowledgment}

This project is supported by Key Laboratory of Micro Nano and Ultra Precision Manufacturing of Jilin Province(No. 20140622008JC), National Natural Science Foundation Item（No. 51375060）.

\section{References}

[1].X.Z. Dong, Q Wei, Z.S. Zhao, et al. Femtosecond pulse laser two-photon micro-nano processing 
technology and its application[J].Chinese Science Bulletin,Vol.53(2008)No.1,p.2-13.

[2].S. Kawata, H.B. Sun, T. Tanaka, et al. Finer features for functional microdevices[J].Nature, Vol.412 (2001),p.697-698.

[3].H.B. Sun, S. Kawata. Two-photon laser precision microfabrication and its applications to micro-nano devices and systems[J].Journal of Lightwave Technology, Vol.21(2003)No.3,p.624 $-633$.

[4].T. Dong, L. Zhu, L.Y. Hou, et al. The smoothing processing of STL model in the field of Laminated Object Manufacturing[J]. Chinese Journal of Mechanical Engineering,Vol.39(200 -03)No.1,p.117-123.

[5].J.B. Zhao, W.J. Liu. Recent progress in slicing algorithm of rapid prototyping technology[J].Computer Integrated Manufacturing Systems, Vol.15(2009)No.3,p.209-221.

[6].S. Zhong, Y.Q. Yang. Precise slicing method based on STL in RE/RP integrated system[J].Computer Intergrated Manufacturing Systems,V0l.18(2012)No.6,p.1145-1150.

[7].J.B. Zhao, W.J. Liu, Y.C. Wang. Smooth technique of STL model intersection contours based on NURBS curves[J].Mini-micro Systems,Vol.25(2005)No.3,p.497-502.

[8].E.T.Y. Lee. Choosing nodes in parametric curve interpolation[J].Computer-Aided Design, Vol.21(1989)No.6,p.363-370.

[9].Y.G. Pi, D.H. Fan. B-Spline curve fitting of consecutive micro-segment trajectory in NC machining[J]. Journal of South China University of Technology(Natural Science Edition),Vol.40

(2012)No.1,p.53-57.

[10].Texas Instruments Inc. Using the DSP BIOS Kernel in Real-Time DSP Applications[Z]. Dallas, Texas: Texas Instruments Inc, 2001,p.02-16. 